\section{PWE-283 SURVEILLANCE FOR HEPATOCELLULAR CARCINOMA: A CLINICAL AUDIT}

doi:10.1136/gutjnl-2012-302514d.283

\section{PWE-284 KHAT AS A POSSIBLE CAUSE OF DRUG INDUCED AUTOIMMUNE HEPATITIS; A CASE SERIES}

doi:10.1136/gutjnl-2012-302514d.284

${ }^{1} \mathrm{~S}$ A Townsend, ${ }^{* 2} \mathrm{C}$ L Ch'ng. 'Department of Gastroenterology, Singleton Hospital, Swansea, UK; ${ }^{2}$ Singleton Hospital, Swansea, UK

Introduction It is thought that mortality due to hepatocellular carcinoma (HCC) is rising in the Western world. This has been primarily attributed to the hepatitis $C$ viral epidemic. ${ }^{1}$ HCC detected after the development of symptoms carries an extremely poor prognosis $(0-10$ per cent survival at 5 years $),{ }^{2}$ whereas those detected at surveillance can often be cured. One study demonstrated reduced mortality of $37 \%$ in cirrhotic patients undergoing 6 monthly surveillance with ultrasound scanning (USS) and $\alpha$ fetoprotein (AFP) levels; this method is currently recommended by the BSG. ${ }^{3}$ However, the American Association for the study of Liver Diseases (AASLD) now advocates 6 monthly USS without AFP testing: studies showed that AFP levels increased detection rate but also increased false positive detection rates with an added cost of $\$ 2000$ to $\$ 3000$ per tumour found. ${ }^{4}$ The clinical lead for hepatology in Swansea, Dr C L Ch'ng, has primarily adopted these guidelines.

Methods A list of 246 patients entered into the HCC surveillance programme between January 2006 and December 2011 was reviewed and the frequency of USS and AFP levels measured for each patient was recorded. 62 patients were excluded:

- 20 DNAs

- 14 new patients

- 13 had insufficient data

- 12 developed serious comorbidities or died before follow-up was complete

- 3 were inappropriate for surveillance

Results were compared with standards set by BSG and AASLD guidelines.

Results 184 patients were appropriate for surveillance

- 183 had at least 1 USS

- 168 had at least 2 USS

- 114 had 6-12 monthly USS

- $95(52 \%)$ had 6 monthly USS (recommended by AASLD)

$48(26 \%)$ received 6 monthly USS and AFP levels (current BSG recommendation).

Conclusion Surveillance of cirrhotic patients for HCC is currently suboptimal, with poor adherence to national guidelines. There is evidence that patients engaged initially but timing of subsequent USS and AFP teasting was erratic. Despite this, results are favourable in comparison with a large US study of cirrhotic hepatitis C carriers which demonstrated routine surveillance in only $12 \%$ of patients. Results did not differ widely from similar departmental audits carried out in the last 5 years. Suggestions for the future include routine 6 monthly postal invitation to screening with facilitated access to scans, together with education of both patients and treating clinicians regarding HCC risk. This should ideally be carried out within an established local surveillance scheme.

Competing interests None declared

\section{REFERENCES}

1. Serag HB, Mason AC. Rising incidence of hepatocellular carcinoma in the United States. N Engl J Med 1999; 340:745-50.

2. Llovet JM, et al. Hepatocellular carcinoma. Lancet 2003;362:1907-17.

3. Ryder SD. Guidelines for the diagnosis and treatment of HCC in adults. Gut 2003; 362:1907-17.

4. Santagostino $\mathbf{E}$, et al. A 6-month versus a 12-month surveillance for hepatocellular carcinoma in 559 haemophiliacs infected with the hepatitis $\mathrm{C}$ virus. Blood 2003;102:78-82
S Riyaz, ${ }^{*}$ M Imran, M Karajeh. Department of Hepatology, NHS, Sheffield, UK

Introduction Khat is well recognised for its hepatotoxic effects. The exact mechanism by which it causes liver damage remains presenting with acute hepatitis, and review the potential role of khat in triggering auto immune hepatitis (AIH).

Methods The database at Sheffield Hospitals was searched for 2010 with liver problems and a history of khat use. They were then assessed for probability of having AIH using the revised criteria for diagnosis of $\mathrm{AIH}^{1}$

Results Six patients presenting with acute hepatitis on a background of khat use were identified. All of the patients were male. Five of these patients were of Somali origin, while one patient was from Yemen. The age range of these patients was 24-57years (mean 42.3 years). The patients were scored according to the revised autoimmune hepatitis criteria. They were given minus four $(-4)$ for khat use on the scoring system due to its potential hepatotoxicity. Despite this, five out of six patients had a pre treatment score of 10 to 15 which placed them in the probable group for autoimmune hepatitis. The five patients that were in the probable group had at least a partial response to corticosteroids with a greater than $50 \%$ reduction in their ALT after 1-month of treatment. The patient that had scored negative for AIH $(<10)$ showed the least improvement with prednisolone and continued to have raised liver enzymes after

Conclusion The exact mechanism by which khat causes hepatotoxicity remains elusive. One possibility could be by triggering autoimmune hepatitis in a genetically susceptible individual. Further studies are needed to evaluate this phenomenon. ${ }^{2} 3$

Competing interests None declared.

\section{REFERENCES}

1. Alvarez $\mathbf{F}$, et al. International Autoimmune Hepatitis Group Report: review of criteria for diagnosis of autoimmune hepatitis. J Hepatol 1999;31:929-38.

2. Chapman $\mathbf{M H}$, Kajihara M, Borges G, et al. Severe, acute liver injury and khat leaves. N Engl J Med 2010;362:1642-4.

3. Peevers CG, Moorghen M, Collins PL, et al. Liver disease and cirrhosis because of Khat chewing in UK Somali men: a case series. Liver Int 2010.

\section{PWE-285 CLINICAL BUT NOT HISTOLOGICAL FACTORS PREDICT LONG-TERM PROGNOSIS IN PATIENTS WITH BIOPSY PROVEN ADVANCED ALCOHOLIC LIVER DISEASE}

doi:10.1136/gutjnl-2012-302514d.285

${ }^{1,2} \mathrm{~S}$ Masson, ${ }^{* 1} \mathrm{I}$ Emmerson, ${ }^{1} \mathrm{E}$ Henderson, ${ }^{2} \mathrm{E}$ Fletcher, ${ }^{2} \mathrm{~A}$ Burt, ${ }^{2} \mathrm{C}$ Day, ${ }^{3} \mathrm{~S}$ Stewart ${ }^{1}$ Liver Transplant Unit, Freeman Hospital, Newcastle, UK: ${ }^{2}$ Institute of Cellular Medicine, Newcastle University Medical School, Newcastle, UK; ${ }^{3}$ Department of Gastroenterology, Mater Misericordiae University Hospital, Dublin, Ireland

Introduction Alcoholic liver disease (ALD) is a significant and increasing threat to the health of the British population. It remains one of the commonest indications for liver transplantation and a leading cause of death. Despite this, the long term clinical course and predictive factors of survival in advanced ALD have not been well described. We aimed to identify factors that predict 15-year survival in out-patients with biopsy-proven advanced ALD.

Methods Patients attending clinic in our institution during early 1996 ( $n=134$ ) with biopsy proven advanced (stage III or IV) ALD were followed-up for 15 years or until death or transplantation. At unknown. We report a series of patients with a history of khat use patients referred to the Hepatology department between 2005 and 1-year of treatment. 